\title{
Reduction of Harmful Pressure Fluctuations in Pipelines by Means of Introduction of Energy Damping Segments
}

\author{
Fedor V. Rekach ${ }^{1}$, Svetlana L. Shambina ${ }^{1_{+}}$and Yuri V. Belousov ${ }^{1,2}$ \\ ${ }^{1}$ Peoples' Friendship University of Russia (RUDN University), 6 Miklukho-Maklaya Street, Moscow, \\ 117198, Russian Federation \\ ${ }^{2}$ Bauman Moscow State Technical University, 5, Baumanskaya 2-ya Street, Moscow, \\ 105005, Russian Federation
}

\begin{abstract}
Problems of water hammer reduction in the pipeline, as well as reduction of the oscillations amplitude of the high pressure wave with a relatively wide front are relevant in our time in the design of pipeline systems, especially for long sections of the pipeline. Dozens of new protective equipment have been developed in the last decade, but interest in the subject has not diminished. This work is devoted to the search for new ideas to achieve the operation safety of modern pipelines. In the article the basic idea of protection is formulated, and also numerical experiments for various schemes of installation of the considered equipment are carried out. The paper provides recommendations on the most appropriate placement of equipment, as well as discusses the issues of economic feasibility.
\end{abstract}

Keywords: hydraulic schemes, pressure fluctuations, water hammer, long pipelines.

\section{Introduction}

Technical progress in the development of pipeline schemes provides for increased requirements for safe operation of the main components of the hydraulic system, including also its linear part. When an increased pressure wave propagates along a long pipeline, plastic deformations [1] may occur, leading to weakening of the strength characteristics of the pipeline system. In case of a sharp increase in pressure, the pipeline may break with severe economic consequences and human casualties. In this regard, scientists continue to search for optimal means of protection from water hammer and unwanted long-term fluctuations in pressure and liquid discharge. The appearance of an obstacle to the movement of the liquid leads to a decrease in flow, and, consequently, an increase in pressure. In this paper, the obstacle is simulated by instant closing of the valve. At the same time, the liquid flow stops and a high pressure wave with a high speed (at least a thousand meters per second) begins to spread along the pipeline. In practice, increase (decrease) in pressure may be related to other factors: the stop of the pumping station work, emergency release of liquid, failure of the controlling system, etc. The basic idea of protection of the pipeline is that the element of the device detecting water hammer transmits the signal to other points of the pipeline, the fluid flow in pipeline is stopped, and the liquid is sent through devious ways with selected hydraulic resistance in the form of perforated apertures which significantly reduce energy of the flow. Perforation serves to control and easy calculation of hydraulic resistances. It is obvious that these devices work well in areas where there is a flow rate.

\section{Main part}

Historical documents and other sources tell us about the construction of bridges and structures associated with rivers on the territory of Russia and the development of water transport, and about the achievements to create devices that would use the water flow energy and other facilities, the operation of which would be im-

\footnotetext{
+ Corresponding author. Tel.: +7 (916) 513-16-98

E-mail address: shambina_sl@mail.ru
} 
possible without knowledge of the basics of hydraulics. It is interesting, that there were pipelines of pottery and wood in Russia in X-XVI centuries; the floating bridge through Dnieper in Kiev was constructed in 1115. In the XIV and XV centuries, water was got from underground sources equipped with modern devices.

Dams that raise the water level were created on the numerous rivers on the European territory of Russia in the middle ages. For example, a stone dam was constructed in the Neglinka river in Moscow in 1625.

The founders of hydraulics and hydraulic structures in Russia were complete members of Academy of Sciences of Russia: M.V. Lomonosov (1711-1732), Daniel Bernoulli (1700-1782) and Leonard Euler (17071783). These scientists laid the foundations of water flows, discovered and tested in practice the laws related to the movement of liquid. The famous Russian scientist N.E. Zhukovsky [2], [3] laid the foundations of hydraulics. He developed the theory of water hammer in the pipeline, and also solved many problems related to water supply, sewerage, and engineering equipment to solve specific problems.

The theory of unsteady motion of a viscous and compressible liquid in pipes was created by I.A. Czarny, [4], who used the hypothesis of quasistationarity, first adopted by S.A. Christiannovich for the calculation of unsteady flow in rivers. The essence of the hypothesis is that the friction force of the liquid against the pipe wall in a non-stationary mode is the same as in a stationary flow at a speed equal to the instantaneous speed of the considered stationary flow. In the book of I.E. Idelchik [5] a wide range of different hydraulic resistances is considered. English scientist D. Fox [6] developed a graphical method for solving problems related to unsteady fluid motion. He laid the foundations of the numerical method of characteristics and developed the main dependences that allow to use different devices in numerical schemes.

Questions of practical application of the equipment, stabilizing pressure in short pipelines were in the works of R.F. Ganiev, H.N. Nizamov and E.I. Derbukov [7]. This problem is relevant in connection with the supply of liquid fuel to rocket engines. Pressure stabilizers can be used in heat supply lines, water supply pipes, sewage systems, as well as in any problems in which the movement of liquid is realized. The resonance was studied in the works of B.F. Glikman [8]. The oscillations in the feed systems of liquid fuel in the rockets are considered in the works [9], [10].

This article focuses on search for a new type of equipment which allows reducing pressure in the event of water hammer or increase (decrease) of pressure due to the other reasons without significant economic costs. Such emergencies are possible due to the failure of the pumping station, the wrong signal of the controlling electronic system, unauthorized release of liquid from the pipeline, etc.

If water stops at once the speed of water $v[\mathrm{~m} / \mathrm{sec}]$ and the velocity of high pressure waves is $c[\mathrm{~m} / \mathrm{sec}]$, there is a water hammer with the value $p=\rho \cdot v \cdot c[\mathrm{~Pa}]$, where $\rho\left[\mathrm{kg} / \mathrm{m}^{3}\right]$ is density of a liquid. The paper considers electrical control of protective equipment, but it is also advisable to duplicate this process by mechanical means as well. The equations of fluid movement and continuity of liquid flow are represented below:

$$
\begin{aligned}
& \frac{\partial}{\partial x}\left(\rho g z+p+\alpha \rho \frac{V^{2}}{2}\right)+\alpha^{\prime} \rho \frac{\partial V}{\partial t}+\frac{\rho \lambda}{2 D} v|v|=0,\left[N / m^{2}\right] \\
& v \frac{\partial p}{\partial x}+\frac{\partial p}{\partial t}+\rho c^{2} \frac{\partial V}{\partial x}=0, \quad\left[N /\left(w^{2} \cdot \text { sec }\right)\right]
\end{aligned}
$$

where $z$ - geodetic mark of a point (m); $p$ - excess pressure in the pipeline (Pa); $\alpha$-Coriolis factor (adopted $\alpha=1) ; v$ - flow speed $(\mathrm{m} / \mathrm{sec}) ; x$ - coordinate along pipe`s axe $(\mathrm{m}) ; \rho$-mass density of liquid (const) $\left(\mathrm{kg} / \mathrm{m}^{3}\right)$; $g$ - acceleration of free fall $\left(\mathrm{m} / \mathrm{sec}^{2}\right) ; \alpha^{\prime}$ - Businesc factor (adopted $\left.\alpha^{\prime}=1\right) ; t$ - time (sec); $\lambda$ - hydraulic resistance coefficient; $D$ - the pipe diameter $(\mathrm{m}) ; c$ - propagation velocity of waves of increased pressure $(\mathrm{m} / \mathrm{sec})$.

Equations (1) - (2) include two partial hyperbolic differential equations. All the parameters follow the marching scheme. It means that they are changing at the next time interval. Boundary condition should be given as water head function of water discharge. It is also possible to specify a single head or flow rate.

For numerical calculation method of characteristics is applied, which is considered in detail in [11], [12], [13]. As the main characteristics of the flow are taken excess water head $H[\mathrm{~m}]$ and liquid discharge $Q$ 
$\left[\mathrm{m}^{3} / \mathrm{sec}\right]$. The numerical program is written in language $\mathrm{C}++$, the graphical interpretation is written in language Marple. The flow of fluid moving at a speed $\mathrm{v}$ can be schematically considered as a chain of heavy (metal) balls connected by springs. Under steady motion, the springs are not stretched (not compressed) and all the balls move at the same speed from the left to the right (Fig. 1). If balls $R$ and $P$ are suddenly stopped (by means of valves), then there will be low pressure at point $\mathrm{R}$ and high pressure at point $P$. As a result, the wave of low pressure (at point $P$ ) will meet the increased pressure wave (from point $R$ ). The area between points $R$ and $P$ will turn into a small-length oscillating circuit with a rapid damping of the pressure's amplitude.

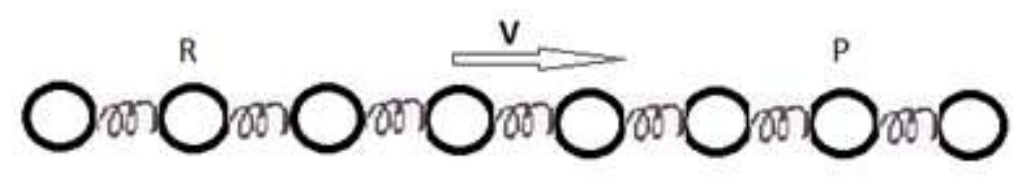

Fig. 1: Scheme of the flow of fluid

In fact, the paper deals with the question of disabling the kinetic energy of the fluid flow [14], [15]. The idea of energy dissipation lies in the fact, that the sharp pressure increase at the point $A$, the electric signal with a propagation speed of electromagnetic fields (up to 300,000 meters per second) is transmitted to other points of the pipeline where the current of the liquid is stopped and the liquid is sent by devious means with a large hydraulic resistance. In this case, the disabling of the kinetic energy of the flow does not start at the point where the wave of increased pressure has already reached, but in areas where it will reach after a while. Thus, the fluid flow is divided into several sections, which creates oscillating processes and, accordingly, decreases the velocity of the liquid. The question of further threat of pressure increase is discussed.

The basic investigated hydraulic scheme is given in Fig. 2. It includes 21 pipes and 18 nodes. The pump is installed in node 1 . Boundary conditions in nodes 18 and 17 are given as constant water heads.

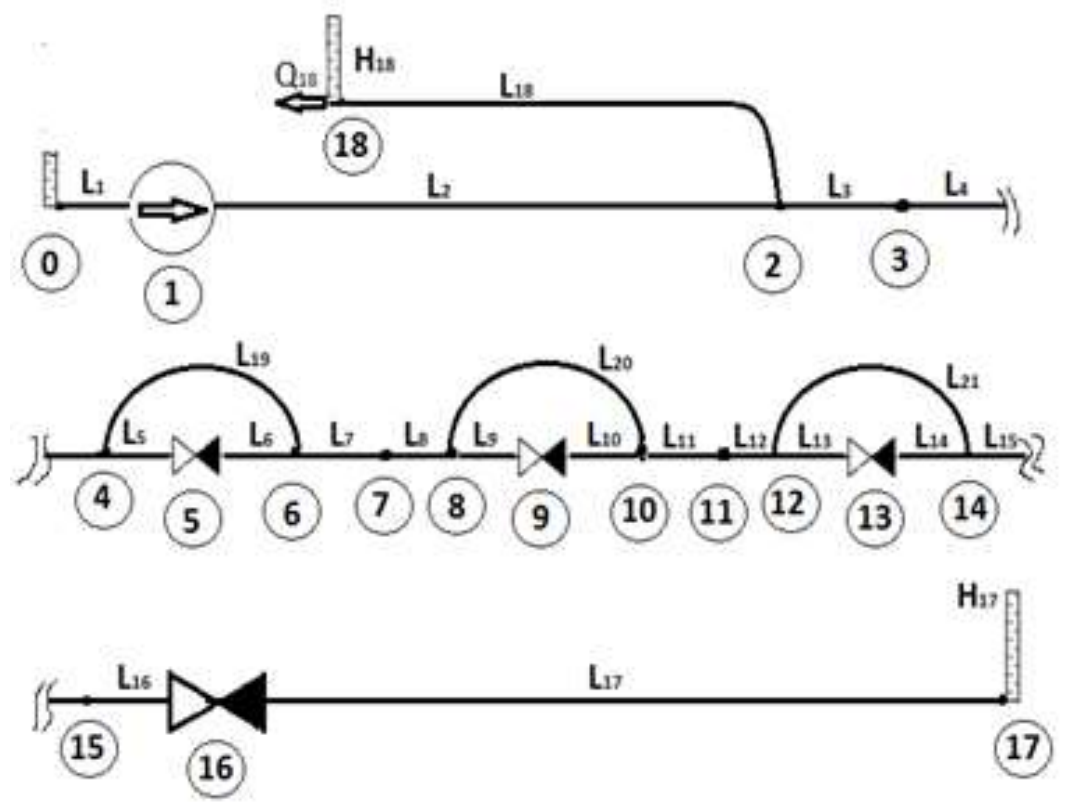

Fig.2: The hydraulic pipeline scheme

A wave of high pressure starts moving from node 16 to node 2 . Then it separates into two flows, moving to points 1 and 18 . In point 16 pressure raises almost instantaneously and reaches its maximum value. Then high pressure wave (if there aren`t any obstacles) moves to points 1 and 18 with speed of $2020 \mathrm{~m} / \mathrm{sec}$. Numerical experiments have one aim: to make braking segments in elements 19, 20 and 21 to decrease significantly the pressure in the system. The devices stopping the flow are installed in nodes 5, 9 and 13 (points $D$, $C$ and $B$ ), they receive a signal to react when the pressure in node 15 (point $F$ ) exceeds the pressure specified in the problem data. 


\section{Numerical Calculations and the Main Results.}

Test problem 1. If consider constant head to be in the node $18\left(H_{18}=60 \mathrm{~m}\right)$, the pressure and discharge in node 15 (point F) is presented in Fig. 3. Maximum pressure (1.85 MPa) is in good coincidence with the theory. We consider, that device, stopping the liquid flow, turns on instantly after time moment when water head exceeds $85 \mathrm{~m}$ in node 15 . The vacuum wave goes from node 7 to node 16 . High pressure wave simultaneously runs from node 16 to node 7 . Pressure oscillation period between nodes 7 and 16 after the addition of these waves is about 9 seconds. This is much less than the oscillation period of the entire system (about 64 seconds) from nodes 11,18 to node 7.

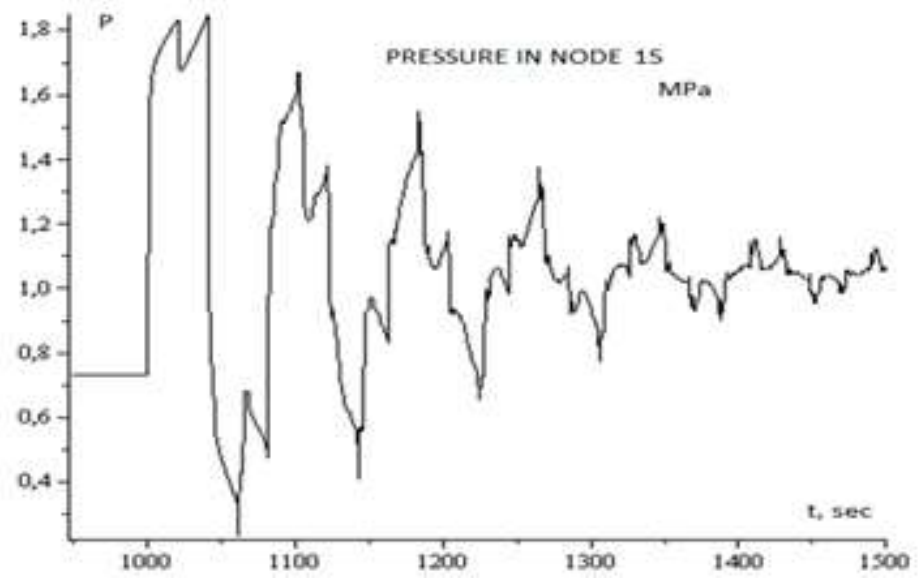

Fig. 3: Pressure at node 15 without energy disabling with water head $H_{18}=60 \mathrm{~m}$

Problem 2. The initial data is changed (in comparison with test problem 1): $\lambda_{20}=0.04, d_{20}=0.1 \mathrm{~m}$. We consider, that device, stopping the liquid flow in node 9 and 16, turns on instantly after time moment when water head exceeds $85 \mathrm{~m}$ in node 15 . Here two waves, running from node 9 to node 16 and vice versa are summed. Oscillation period in node 15 (as well as from node 9 to node 16) is two times less than water head $H_{9}=$ const (Fig. 4). Peaks of the maximum pressures are less. This is also a positive fact. It is interesting fact that with a decrease of the friction coefficient in the pipe 20 (or an increase in the pipe diameter in tube 20), the pressure even rises after some time passes. This can be due to the influence of flow to the left of node 8 .

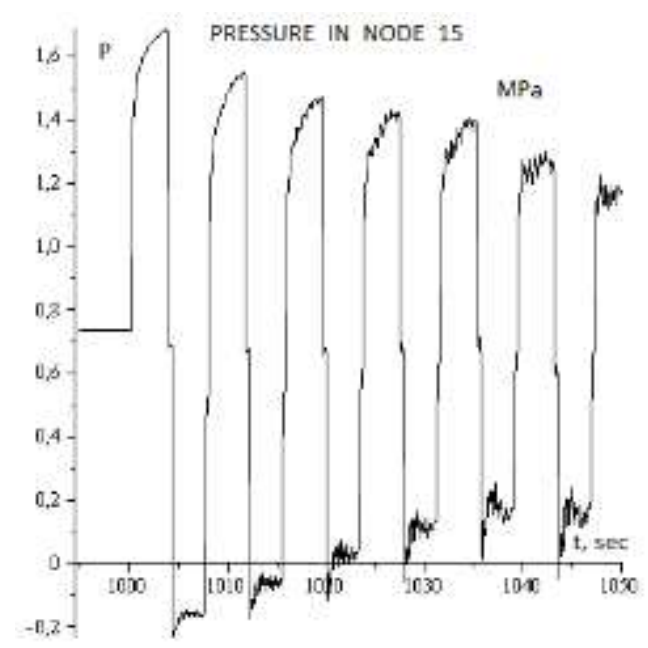

Fig. 4: Pressure in node 15 when the valve in node 9 $\left(t_{c l}=0\right)$ closes from a signal of node 15 .

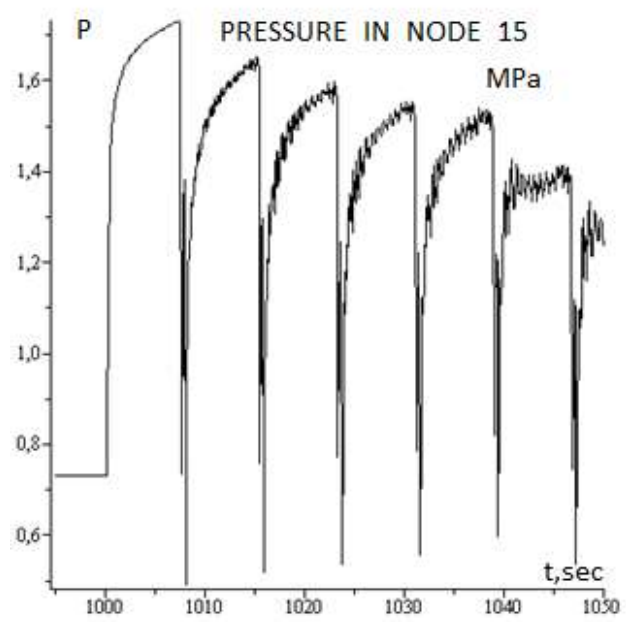

Fig. 5: Pressure in node 15 when the valve in node 9 is actuated $\left(t_{c l}=0\right)$ from the head increase in node 9 up to $85 \mathrm{~m}$.

Problem 3. It differs from problem 2 that the valve in node 9 closes when the pressure in node 9 exceeds $85 \mathrm{~m}$ (Fig. 5). It is interesting that there is no vacuum wave from node 9 and therefore the average pressure is much higher than in problem 2. Besides, high pressure peaks are wider. All this suggests that it is more difficult to extinguish the overpressure in this case than in the example of problem 2. 
Problem 4. It differs from the problem 2 is that the valves in the nodes $5,9,15$ close when the pressure in the node 15 exceeds $85 \mathrm{~m}$ (Fig. 6, 7). In the problem the oscillation frequency is high in node 15 (Fig. 6), because the distance between node 13 and 16 is not large $(600 \mathrm{~m})$. This creates good conditions for breaking the flow. It is important to note that the pipeline from node 1 to node 5 is poorly protected, but the period of pressure fluctuations in node 3 is less than without electrical devices.

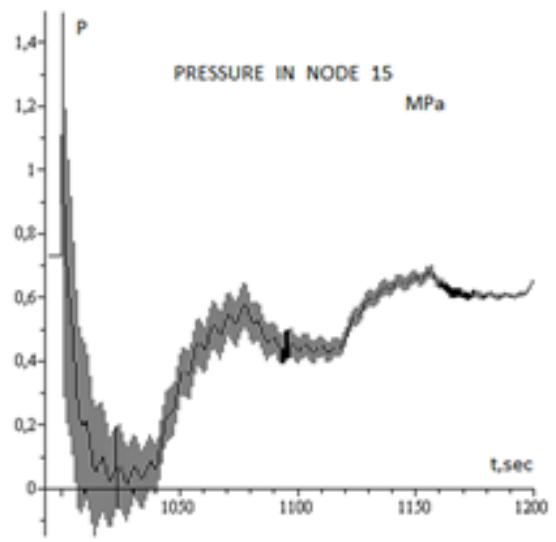

Fig. 6: Pressure in node 15 when devices in nodes 5, 9,13 close $\left(t_{c l}=0\right)$ from pressure increase in node 15 over $85 \mathrm{~m}$.

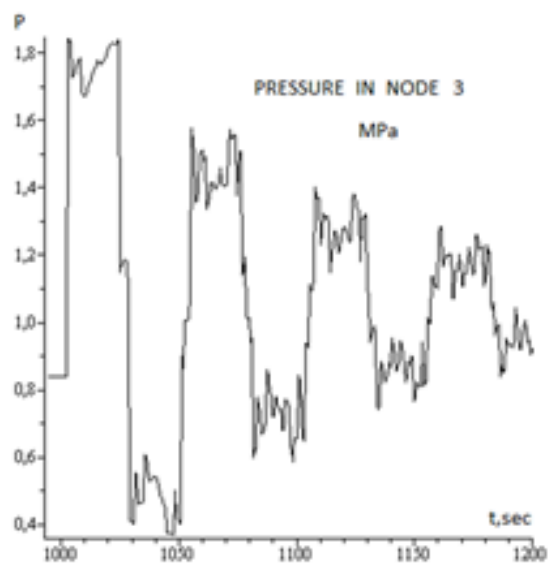

Fig. 7: Pressure in node 3 when devices in nodes 5, 9,13 close $\left(t_{c l}=0\right)$ from pressure increase in node 15 over $85 \mathrm{~m}$.

\section{Conclusions}

1) Water flow rate should be used very carefully as a boundary condition. As shown by the analyses, as closer this boundary condition to the border then the less accurate is the calculation. In the results we can obtain higher values of pressure.

2) Introduction of devices, closing almost simultaneously creates a wave of vacuum. It is summing with a wave of high pressure. As a result, the pressure at the node 15 is significantly less than without them (Fig. 3 ). The period of pressure fluctuations is two times less, so it is much easier to extinguish such oscillations than the oscillations of all systems.

3) The devices, closing simultaneously, divide the water flow into shorter sections. Here the mileage of high pressure and vacuum waves is correspondingly less. This greatly facilitates the fight against the increased pressure. Periods of pressure fluctuations are different (64 sec and $82 \mathrm{sec}$ ).

4) Introduction of self-actuating valves when pressure increases has a higher average pressure and wider peaks of high pressure, which makes it difficult to stabilize (equalize) the pressure.

5) Pressure Diagram in Fig. 6 shows that the introduction of three breaking circuits effectively dissipates kinetic energy. Creating of short circuits dissipates kinetic energy well, due to frequent fluctuations of water discharge.

6) Pressure waves with a short period can be well disabled by means of pressure stabilizers.

\section{Acknowledgements}

This paper was financially supported by the Ministry of Education and Science of the Russian Federation on the program to improve the competitiveness of Peoples' Friendship University of Russia (RUDN University) among the world's leading research and education centers in the 2016-2020. This publication was prepared with the support of the "RUDN University Program 5-100".

\section{References}

[1] M. Rynkovskaya. Plastic deformations occurring in shells with developable middle surfaces during bending IOP Conf. Series: Materials Science and Engineering. 2018, 371: 012054 
[2] N.E. Zhukovsky. Collected works in 7 volumes. M.; L.: Gostekhizdat, 1949. Volume 2: Hydrodynamics - 763 p.; Volume 3: Hydraulics. Applied mechanics. - 700 p.

[3] N.E. Zhukovsky. On Water Hammer in Water Pipes. M.-L.: Gostehizdat, 1949.

[4] I.A. Charny. Unsteady movement of a real liquid in pipes. M.: Nedra, 1975.

[5] I.E. Idelchik. Reference book on hydraulic resistance. Moscow: Mechanical engineering, 1975.

[6] D.A. Fox. The hydraulic analysis of the unsteady current in pipelines . M, Energoizdat, 1981.

[7] R.F. Ganiev, H.N. Nizamov, E.I. Derbukov. Wave Stabilization and Prevention of Accidents in Pipe-Lines. Moscow: Izd-vo MGTU, 1996.

[8] B.F. Glikman. Mathematical models of pneumohydraulic systems. M., Nauka, 1986.

[9] K.S. Kolesnikov. Longitudinal oscillations of a liquid-propellant rocket engine. M.: Mashinostroenie, 1971.

[10] M.S. Natanzon. Longitudinal self-oscillations of a liquid rocket. M.: Mashinostroenie, 1977.

[11] F.V. Rekach, S.L. Shambina, E.K. Sinichenko. Influence of pressure stabilizer perforation area on character of unsteady fluid motion in hydraulic systems. International Journal of Mechanical Engineering and Robotics Research. 2017, Vol. 6 (4): 268-271.

[12] F.V. Rekach. Calculation of fluctuations in circular cylindrical shells with pressure stabilizer by method of characteristics. Structural Mechanics of Engineering Constructions and Buildings. 2010, 1: 60-65.

[13] F.V. Rekach, S.L. Shambina, Yu.V. Belousov, Fibor J. Tan. Water Hammer Decrease by Introduction of Pipes with Low Speed Pressure Waves Propagation. J. Mech. Cont. \& Math. Sci. 2019, Sp.Iss.1: 671-682.

[14] W.R. Young and C.L. Wolfe. Generation of surface waves by shear-flow instability. J. Fluid Mechanics. 2014, Vol. 739: 276-307.

[15] Samuel S. Pegler. The dynamics of confined extensional flows // J. Fluid Mechanics. Vol. 804. - P. 24 - 57 (2016).

[16] Pietro Scandura, Carla Faraci, Enrico Foti. A numerical investigation of acceleration-skewed oscillatory flows. $J$. Fluid Mechanics. 2016, 808: 576-613. 